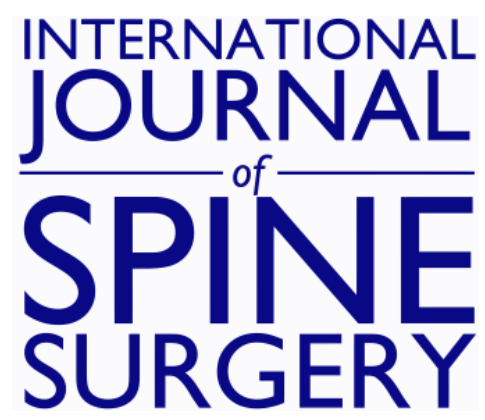

\title{
Delayed Incisional Hernia Following Minimally Invasive Trans-Psoas Lumbar Spine Surgery: Report of a Rare Complication and Management
}

MUKUND GUNDANNA and KUNAL SHAH

Int J Spine Surg 2018, 12 (2) 126-130

doi: https://doi.org/10.14444/5019

http://ijssurgery.com/content/12/2/126

This information is current as of April 26, 2023.

Email Alerts Receive free email-alerts when new articles cite this article. Sign up at: http://ijssurgery.com/alerts 


\title{
Delayed Incisional Hernia Following Minimally Invasive Trans-Psoas Lumbar Spine Surgery: Report of a Rare Complication and Management
}

\author{
MUKUND GUNDANNA, MD, KUNAL SHAH, MS \\ Brazos Spine, College Station, Texas
}

\begin{abstract}
Background: Lateral lumbar interbody fusion (LLIF) has proved to be a safe tool in the armamentarium of spine surgeons for a variety of lumbar disorders. However, it has some complications related to specific approaches. Incisional hernia following abdominal surgery and anterior spinal surgery is commonly described; however, it is extremely rare following LLIF, with only 1 case reported in short postoperative period. In this report we present the first case of delayed presentation of true incisional hernia following a LLIF procedure and highlight its presentation, mechanism, possible preventive measures, and management.

Methods: We report a 57-year-old lady who underwent L3-4 LLIF. She presented with vague pain in a healed scar area that had no swelling until two years postsurgery, when she developed a painful swelling. On examination, it appeared to be a herniation of abdominal contents.

Results: She underwent a laparoscopic hernia repair surgery. The muscular layers were found to be intact with an attenuated transversalis fascia layer. The repair was reinforced by polypropylene mesh. There was no recurrence at the 6-month follow-up.

Conclusions: Incisional hernia can occur following LLIF months to years following surgery and can have varied presentation. Tight external oblique closure should be performed because the transversalis fascia often cannot be repaired and the quality of a layered closure of the deep obliques is often disappointing. The treating surgeon should be aware of this complication and aggressively surveil for the warning signs, and patients should be counseled about this potential complication.
\end{abstract}

Complications

Keywords: lateral lumbar interbody fusion, incisional hernia, transversalis fascia

\section{INTRODUCTION}

Minimally invasive spine (MIS) surgery has become increasingly popular over the last decade with its advantages of having a shorter postoperative recovery time and relatively lower complication rates. ${ }^{1}$ MIS surgeries like anterior lumbar interbody fusion (ALIF), lateral lumbar interbody fusions (LLIF), percutaneous transforaminal interbody fusion, etc, are routinely performed for lumbar degenerative pathologies, which are commonly seen. ${ }^{2,3}$ With each new procedure performed, different types of complications are encountered. LLIF is popularly used amongst spine surgeons for a variety of lumbar disorders. It is a safe procedure; however, a variety of complications are associated. Here we report the occurrence of a rare complication: an incisional hernia that presented two years following lateral lumbar interbody fusion. We will highlight its possible etiology and possible preventive measures with its management.

\section{CASE REPORT}

A 57-year-old female presented with severe back pain and left lower limb radiculopathy and tingling numbness, worsening over 3 years. She had not responded to nonoperative management. She was medically healthy and a nonsmoker. On examination she had local tenderness over the L3-4 region with positive straight-leg raise tests. Spinal range of motion was restricted. Motor examination suggested left ankle dorsiflexion 3/5. Her radiographs of the lumbar spine showed reduced disc space at L3-4 with kyphosis and subtle translational instability. Her magnetic resonance image showed degenerative disc disease with stenosis at the L3-4 level with facet arthropathy and Modic changes (Figure 1). She also 


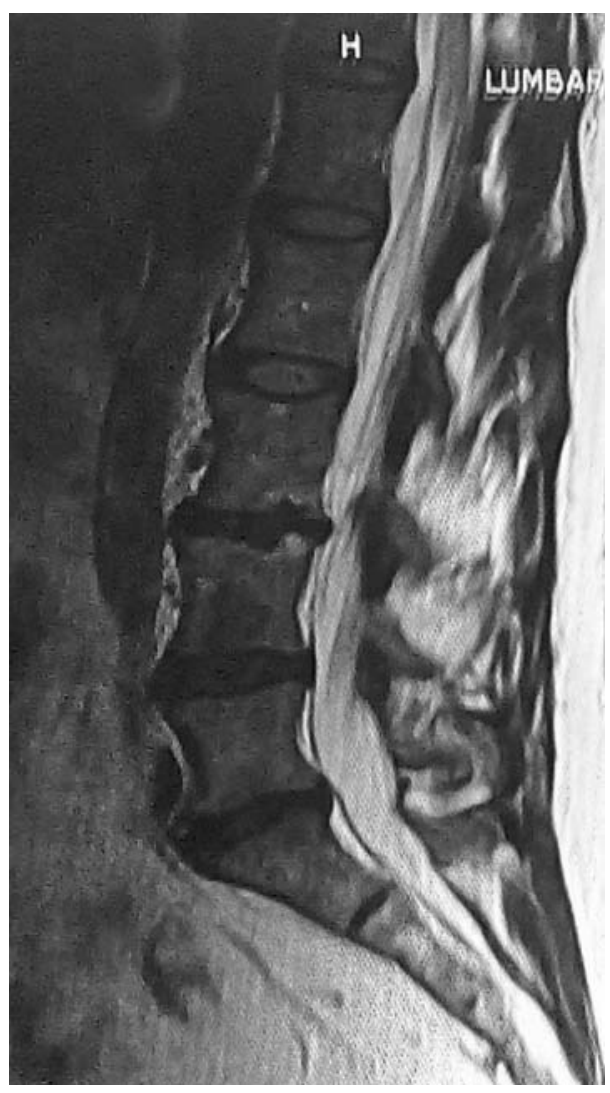

Figure 1. Magnetic resonance image showing lumbar degenerative disc disease with L3-4 stenosis.

had some degeneration at L4-5 and L5-S1. To ensure that the L3-4 level was the main pain generator, provocative discography was done with proximal and distal controls, confirming the L3-4 level as the primary pathology. We offered her either a minimally invasive transforaminal lumbar interbody fusion or a minimally invasive direct lumbar interbody fusion with posterior interspinous plating. The LLIF and plating were chosen.

\section{SURGERY}

Standard transpsoas approach was performed for lateral interbody fusion. After general anesthesia, the patient was positioned in the right lateral decubitus position (left side up). Levels were checked under fluoroscopy and a horizontal $5-\mathrm{cm}$ skin incision was taken along Langer's lines. Subcutaneous tissue was sharply divided, and the fascia with oblique muscles were visualized. Sharp dissection was used to divide the external oblique fascia, and the internal and innermost obliques were divided with finger dissection along their fibers. Following this, the transversalis fascia was encountered. This was bluntly dissected with finger dissection to reach the retroperitoneal space. The retroperitoneal fat was mobilized bluntly anteriorly, and the transverse processes were palpated. The psoas was then bluntly palpated, along with the anterior and posterior margins of the vertebral body. Using finger dissection, the psoas was split along natural planes, down to the disc level. After the surgeon felt the crest of the disc space, the initial dilator was slid behind the finger, down to the psoas defect. The final retractor size was determined and placed over the initial dilator and secured to the table. Fluoroscopy and neuromonitoring were used to confirm the position of the final retractor. Probes were used to open the psoas split, and the Clowards were placed as well as the inner-ring cannula. Neuromonitoring was stable.

Discectomy and interbody fusion were done with standard techniques, ensuring that the far annulus was released. A $9 \mathrm{~mm}$ by $17 \mathrm{~mm}$ by $40 \mathrm{~mm}$ cage was placed (Veo, Choice Spine) after being packed with bone graft. Position was confirmed under both antero-posterior and lateral fluoroscopy. After irrigation, the retrators were removed and soft tissue planes reapproximated. As is very common, the transversalis fascia was attenuated and stretched and was not closed. For closure, the external oblique fascia was closed tightly with figure-of- 8 stitches. The skin was tightly closed with buried 3-0 Vicryl stitches. The patient was then turned to a prone position, and a midline approach was taken to place an interspinous plate (Lanx straight plate) with a ligament-sparing technique. There were no adverse intraoperative and postoperative events, and $\mathrm{EBL}$ was $<50 \mathrm{~cm}^{3}$.

\section{POSTOPERATIVE COURSE}

Patient was mobilized as tolerated in the postoperative period and discharged on the first postoperative day. Postoperative radiographs showed wellplaced implants, and the patient reported significant relief from symptoms. The wound healed well at 14 days postoperatively. The patient was advised to stay away from unnecessary twisting and bending for three weeks to allow for soft tissue healing and closure. At 3 months, the patient was doing very well and was back to work with no restrictions. However, she complained of vague pain at the lateral incision at the 6-month follow up. There were no significant examination findings apart from some dull tenderness to deep palpation in the surgical area. Two years postsurgery, the patient noticed a 


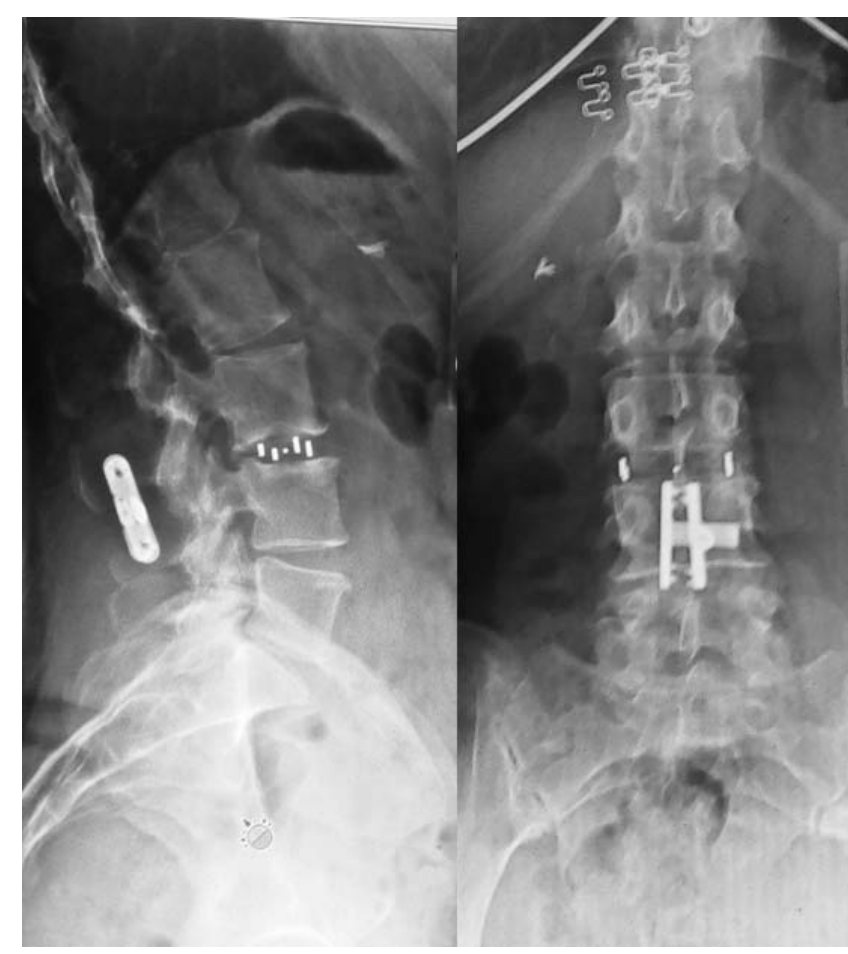

Figure 2. Radiographs (anteroposterior and lateral view) showing well-placed implants.

painful swelling at the surgical site that would grow larger with coughing. She did not perform any recent unusual strenuous activity. There were no associated constitutional symptoms suggestive of infection. On examination, the size of the swelling was $6 \mathrm{~cm}$ by $8 \mathrm{~cm}$ and globular. It was soft on palpation and easily reducible. The size increased with valsalva. Radiographs showed well-placed implants (Figure 2). Her hernia was photographed (Figure 3). She was promptly referred to a general surgeon for evaluation of her likely hernia. Based on clinical findings, the diagnosis of incisional hernia was made, and laparoscopic hernia repair was performed the following day. Figure 4 shows intraoperative pictures of herniated bowel (Figure 4a) and repair reinforced with mesh (Figure $4 b)$.

\section{OPERATIVE FINDINGS}

Through a laparoscopic portal, a $2 \mathrm{~cm}$ by $2 \mathrm{~cm}$ defect was visualized in the transversalis fascia. The fibers of the obliques could be seen through the hernia window. The closure was performed with polypropylene mesh secured with staples and stitches (Figure 3). The tone of the abdominal wall in the hernia region was found to be normal. There were no atrophic or atonic changes. The patient's lateral abdominal pain improved postoperatively.

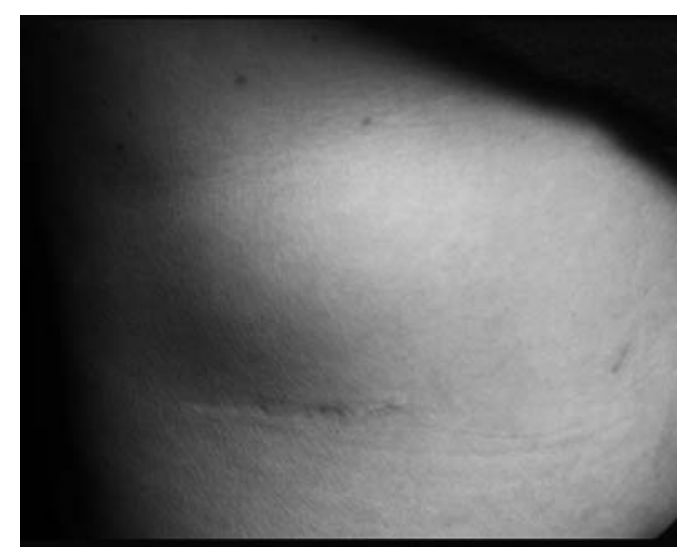

Figure 3. Clinical picture showing globular swelling in healed surgical site area.

There has been no recurrence of the hernia or any abdominal symptoms after 6 months. The patient is back to unrestricted activity.

\section{DISCUSSION}

Lateral lumbar interbody fusion, also known as extreme lateral interbody fusion or direct lateral interbody fusion, is performed in the lumbar and thoracic spine for various conditions like degenerative disc disease, stenosis, low-grade spondylolisthesis, degenerative scoliosis, and infection. It is also known as the transpsoas approach in the lumbar spine because it traverses through the psoas muscle. ${ }^{4,5}$ The potential benefits of the lateral approach are the avoidance of lumbar muscle dissection and dura and nerve root injury (common in posterior and transforaminal approaches), and mobilization and retraction of the iliac tree (needed in anterior approaches). ${ }^{4,6}$ Thus it is a valuable tool in the armamentarium of spine surgeons.

There are some unique complications attributed to this approach. These include injury to nerves passing through the psoas muscle (lateral cutaneous nerve, genitofemoral nerve, lumbosacral plexus), which can be transient or permanent, and psoas or quadriceps palsy. ${ }^{4,7}$ Recently visceral injuries like bowel injury, ${ }^{8}$ urethral injury, and kidney injury ${ }^{9}$ requiring surgical intervention were reported in the literature. Incisional hernia is common after abdominal surgery and anterior spine surgeries (including ALIF); however, it is rare with LLIF, with only 1 case reported in the literature. ${ }^{3,10}$

Lateral incisional hernias can occur through two mechanisms. First, an incisional hernia can occur through outpouching of denervated abdominal layers. These hernias are very difficult to repair 

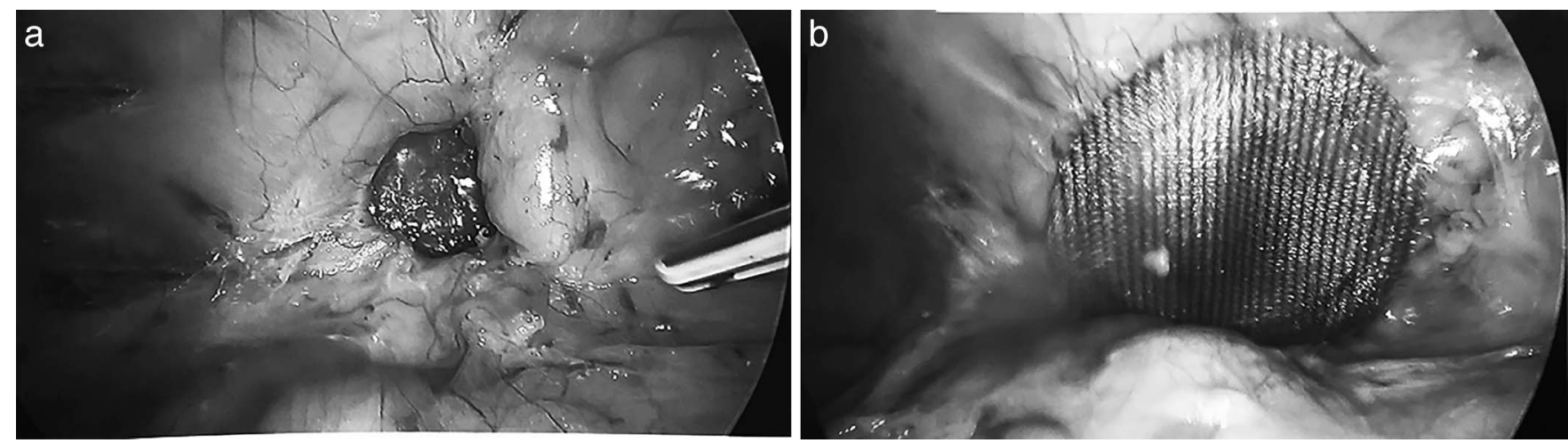

Figure 4. (a) Herniated bowel through defect of transversalis fascia. (b) Hernia repair reinforced by polypropylene mesh.

due to incompetence of the entire muscular wall. ${ }^{11}$ The second type would be a focal herniation through a wall defect in the presence of normal abdominal muscular tone. ${ }^{3}$

Hernias occurring months to years after surgery are described following a retroperitoneal approach performed for aortic aneurysm surgeries ${ }^{12}$ and renal surgeries. ${ }^{13}$ The likely explanation for delayed hernia is the atrophy of muscles following iatrogenic denervation. ${ }^{14}$ The muscles lose integrity following denervation and atrophy, and the wall in that region allows for the herniation. Hence blunt and careful dissection of muscular layers to prevent denervation and subsequent atrophy is recommended. The MIS LLIF is performed with mini incision and minimal handling of the muscular layer, thus minimizing chances of denervation. There is not much denervation with this MIS approach. However, there are recent reports of abdominal pseudohernias following LLIF. Dakwar ${ }^{11}$ and Plata Bello ${ }^{15}$ suggest that direct surgical trauma during dissection of abdominal layers and retroperitoneum could cause injury to motor nerves supplying the abdominal muscles, causing paresis. They also concluded that the paresis resolves within 6 months. Our case presented with vague pain at the healed surgical site at the 6-month follow-up; this can possibly differentiate it from pseudoparesis or neuropraxia. Additionally, on repair of the defect, the abdominal wall had full tone and muscular integrity. All clinical evaluations from the time of surgery until the repair showed no loss of muscular wall tone.

Galan et $\mathrm{al}^{3}$ reported the only case of true incisional hernia following an L4-5 LLIF in a 74year-old female. They reported this complication 4 weeks postoperatively. They concluded that increased intra-abdominal pressure along with weak- ened fascia caused herniation of the bowel. They cited a few recommendations to prevent this complication:

1. Placing the surgical incision posteriorly where the transversalis fascia is thick.

2. Direct repair of transversalis fascia with nonabsorbable suture.

3. Blunt dissection to prevent muscle denervation and atrophy.

4. Aggressive bowel care program to avoid strain causing increased intra-abdominal pressure. ${ }^{3}$

In our case, the onset of the hernia was insidious, with the initial complaints being vague lateral abdominal pain with a benign examination. We suspect that the attenuated transversalis fascia eventually weakened at the incision to a point that abdominal contents could not be contained. Based on laparoscopic imaging, the defect was punctate, with very well-defined margins. The oblique layers were intact and healthy, and the herniation did not breach the muscle layers. The diagnosis of incisional hernia is usually straightforward based on physical findings, as was the case here. Further imaging studies, such as computed tomography (CT), were not required. If the diagnosis was not clear, a CT scan in Valsalva that was described by Jaffe et $\mathrm{al}^{16}$ would have been considered.

Early diagnosis is of prime importance in the case of incisional hernias. Herniated contents are at risk of incarceration and strangulation. They also cause abdominal wall fibrosis, overlying skin changes and bad cosmesis. ${ }^{17}$ Therefore they should be treated aggressively even if the patient is mildly symptomatic or asymptomatic. Our case presented with vague pain with no palpable mass, long before the swelling appeared. Thus this type of presentation 
can delay the diagnosis and it should be kept in mind.

Thus we conclude that incisional hernia can occur following LLIF months to years following surgery. The presentation can range from vague localized pain to an obvious painful mass. Utmost care should be taken while performing surgery to prevent weakening of the musculofascial layers. Tight external oblique closure should be performed because the transversalis fascia often cannot be repaired and the quality of a layered closure of the deep obliques is often disappointing. The treating surgeon should be aware of this complication and aggressively surveil for the warning signs, and patients should be counseled about this potential complication.

\section{REFERENCES}

1. Kim CW, Siemionow K, Anderson DG, Phillips FM. The current state of minimally invasive spine surgery. Instr Course Lect. 2011;60(6):353-370.

2. Spoor AB, Öner FC. Minimally invasive spine surgery in chronic low back pain patients. $J$ Neurosurg Sci. 2013;57(3):203-218.

3. Galan TV, Mohan V, Klineberg EO, Gupta MC, Roberto RF, Ellwitz JP. Case report: incisional hernia as a complication of extreme lateral interbody fusion. Spine $J$. 2012;12(4):e1-e6

4. Kwon B, Kim DH. Lateral lumbar interbody fusion: indications, outcomes, and complications. J Am Acad Orthop Surg. 2016;24(2):96-105.

5. Arnold PM, Anderson KK, McGuire RA Jr. The lateral transpsoas approach to the lumbar and thoracic spine: a review. Surg Neurol Int. 2012;3(suppl 3):S198-S215.

6. Patel VC, Park DK, Herkowitz HN. Lateral transpsoas fusion: indications and outcomes. Sci World J. 2012;2012:893608.

7. Uribe JS, Deukmedjian AR. Visceral, vascular, and wound complications following over 13,000 lateral interbody fusions: a survey study and literature review. Eur Spine J. 2015;24(suppl 3):386-396.

8. Balsano M, Carlucci S, Ose M, Boriani L. A case report of a rare complication of bowel perforation in extreme lateral interbody fusion. Eur Spine J. 2015;24(suppl 3):405-408.

9. Anand N, Baron EM. Urological injury as a complica- tion of the transpsoas approach for discectomy and interbody fusion. J Neurosurg Spine. 2013;18(1):18-23.

10. Paik NC. Incisional hernia after anterior lumbar interbody fusion. Spine J. 2010;10(9):844.

11. Dakwar E, Le TV, Baaj AA, et al. Abdominal wall paresis as a complication of minimally invasive lateral transpsoas interbody fusion. Neurosurg Focus. 2011;31(4):E18.

12. Matsen SL, Krosnick TA, Roseborough GS, et al. Preoperative and intraoperative determinants of incisional bulge following retroperitoneal aortic repair. Ann Vasc Surg. 2006;20(2):183-187.

13. Chatterjee S, Nam R, Fleshner N, Klotz L. Permanent flank bulge is a consequence of flank incision for radical nephrectomy in one half of patients. Urol Oncol. 2004;22(1):36-39.

14. Yamada M, Maruta K, Shiojiri Y, et al. Atrophy of the abdominal wall muscles after extraperitoneal approach to the aorta. J Vasc Surg. 2003;38(2):346-353.

15. Plata Bello J, Roldan H, Brage L, Rahy A, GarciaMarin V. Delayed abdominal pseudohernia in a young patient after an LLIF procedure: case report. World Neurosurg. 2016;91:671.e13-671.e16.

16. Jaffe TA, O'Connell MJ, Harris JP, et al. MDCT of abdominal wall hernias: is there a role for Valsalva's maneuver? AJR Am J Roentgenol. 2005;184(3):847-851.

17. Nieuwenhuizen J, Halm JA, Jeekel J, Lange JF. Natural course of incisional hernia and indications for repair. Scand J Surg. 2007;96(4): 293-296.

Disclosures and COl: There is no conflict of interest. There is no financial assistance gained from this article.

Corresponding Author: Kunal Shah, MS (Orthopaedics), Spine surgery fellow, Brazos Spine, 3526 Longmire, Suite 101, College Station, TX 77845. Phone: +91-9930731911; Fax: (979)-6934706; Email: orthokunal@yahoo.com.

Published 3 August 2018

This manuscript is generously published free of charge by ISASS, the International Society for the Advancement of Spine Surgery. Copyright (C) 2018 ISASS. To see more or order reprints or permissions, see http://ijssurgery.com. 